\title{
Cutaneous nerve stimulation and motoneuronal excitability: I, soleus and tibialis anterior excitability after ipsilateral and contralateral sural nerve stimulation
}

\author{
P J D E L W I DE, P CRENNA, * A N M H FLERON \\ From the Section of Neurology and Clinical Neurophysiology, Department of Internal Medicine, \\ University of Liège, Belgium
}

S U M MARY Modification of soleus and anterior tibial anterior horn cell excitability following ipsilateral and contralateral stimulations of the sural nerve was studied by either the $\mathrm{H}$ reflex (for the soleus and anterior tibial muscles) or the $\mathrm{F}$ response (for the anterior tibial muscles). Several intensities of stimulation were employed. In every instance the recovery curves showed two distinct peaks of facilitation, which appeared with the same delay in muscles with antagonist functions. Also, reciprocal facilitation and inhibition phenomena which occurred after a $25 \mathrm{~ms}$ delay and which lasted more than $1000 \mathrm{~ms}$ were observed. The intervention of suprasegmentary neuronal mechanisms is proposed to explain the facilitation peaks, while the longer lasting phenomena are probably dependent on spinal processes.

In man, the role of exteroceptive afferents in motor control has been analysed by various methods. The first has consisted of testing, in the absence of voluntary contraction, motoneurone excitability by means of its monosynaptic reflex and of studying the recovery curves following electrical stimulation of a sensory nerve. The soleus Hoffman reflex particularly has been used as it is considered a good measure of motoneurone excitability. ${ }^{12}$ The second technique has employed elicitation of flexor reflexes. ${ }^{34}$ The third has consisted of a study of the changes in voluntary activity following graduated stimulation of sensory nerves. ${ }^{5-8}$

Despite these earlier investigations, a number of questions remain unanswered. Even limiting consideration to excitability curves, there are differences not only in the techniques used but also in the results obtained. With the exception of Hugon $^{9}$ no investigators have systematically studied the effects produced both by low intensity

Address for reprint requests: Dr PJ Delwaide, Hôpital de Bavière, 4020 Liège, Belgium.

*Instituto Neurologico, “C Besto", Milano, Italy.

Accepted 18 May 1981 (non-painful) and high intensity (painful stimuli). The spinal afferents activated in these two conditions are different because electrical stimulation recruits fibres of different calibre which transmit impulses at different velocities. In some cases, experimental findings concern only very brief intervals ${ }^{10}$ or are derived from very restricted regions of the body, for example, a single motor nucleus or stimulation of an isolated sensory nerve. Systematic study of two motor nuclei with antagonistic function has not so far been undertaken. For all these reasons, it remains difficult to define clearly in man the functional role of exteroceptive afferents in motor control, and to understand the physiological mechanisms which are involved following stimulation of a sensory nerve. The results of most earlier studies performed in man have been interpreted by mechanisms utilising polysynaptic spinal circuits. ${ }^{11}$ New experimental findings indicate that proprioceptive afferents can modulate motor excitability through suprasegmental, cortical or sub-cortical, circuits. ${ }^{12-16}$ The latency of the effects observed after exteroceptive stimulation raises the question of the role of supraspinal structures in their genesis, in terms of the long loop concept. 
In the present work, we have investigated the excitability of the antagonistic soleus and tibialis anterior motor nuclei following electrical stimulation of the purely sensory sural nerve. The excitability of the soleus and tibialis anterior motoneurone pool were tested by means of the Hoffmann $(\mathrm{H})$ reflex. The $\mathrm{H}$ reflex is inconstant for the tibialis anterior; when it could not be elicited, the $\mathrm{F}$ response was used. ${ }^{1718}$ Three intensities of stimulation were systematically employed: a threshold stimulus, a stimulus giving rise to a sensation of touch, and a painful stimulus. We have studied the changes induced during one second after the conditioning stimulus by exploring a large number of intermediate delays. Finally, we have compared the effects of identical stimuli applied to the ipsilateral and contralateral sural nerves respectively.

\section{Subjects and methods}

Twenty-five volunteer subjects of both sexes, aged between 14 and 30 years (average 22), free from any neurological disorder, were studied-some of them several times. The height of the subjects was measured; they were seated in an examination chair specially adapted for reflex testing of the flexed lower limbs, with the foot fixed in a special holder. The lower leg made an angle of $110-120^{\circ}$ with the thigh and $120^{\circ}$ with the foot. The slightly extended head rested on a headrest, with the eyes open. The subjects were in a room maintained at constant temperature and with low lighting; examinations were carried out in silence. Electrical activity in the soleus muscle was recorded by skin electrodes fixed in the midline of the calf 10.0 and $11.5 \mathrm{~cm}$ above the calcaneum. For the tibialis anterior, the electrodes were fixed over the motor point and a point $2.0 \mathrm{~cm}$ below respectively. Potentials were amplified by a plug-in Tektronix 3A9, then visualised on the screen of a Tektronix 565 oscilloscope. Potentials were measured peak-to-peak by a Tektronix 230 Digital Unit and numerical values were transcribed by a Hewlett Packard printer. Stimulation was by a Grass S88 through constant current isolation units.

The soleus Hoffmann reflex was elicited by a $1 \mathrm{~ms}$ shock applied to the posterior tibial nerve in the popliteal fossa, observing the precautions described in Desmedt. ${ }^{19}$ Reflex contraction took place under isotonic conditions. The value $\mathrm{H} \max / 2$ was taken as test. The monosynaptic reflex in the tibialis anterior was elicited only in seven subjects by inframaximal stimulation of the peroneal nerve at the head of the fibula (1 ms shock). The evoked response was always of small amplitude (on average $100 \mu \mathrm{V}$ ), and the ratio H max/M Max also was small. When expressed as a percentage of control values, facilitations of amplitude reached high values because the reference levels were low. When the $\mathbf{H}$ reflex of the tibialis anterior could not be elicited, the $F$ response was evoked by a supramaximal shock applied to the same site.

The sural nerve was stimulated at the level of the external malleolus by Disa cutaneous electrodes $1.0 \mathrm{~cm}$ apart, ipsilateral or contralateral to the lower limb in which the $\mathrm{H}$ or $\mathrm{F}$ responses were elicited. Placements were sought which elicited a sensation of touch on the outer border of the foot at the lowest intensities of stimulation. Unless otherwise stated, stimulation consisted of a train of seven 1-ms shocks at $300 \mathrm{~Hz}$. In some experiments, the number of shocks varied between one and 12 . The stimulus intensity which elicited a feeble but perceptible tactile sensation was regarded as threshold. Subsequently, the intensity was increased until a clearly tactile sensation was evoked; this usually was two or three times the threshold value. Finally, stimuli evoking frank pain were applied; they were generally above three times the threshold. Sural stimuli were repeated in random fashion over 7.5 to $9.0 \mathrm{~s}$. From the beginning of each stimulus sequence (time 0 ), electric shocks evoking an $\mathrm{H}$ or $\mathrm{F}$ response were applied after delays usually increasing by $10 \mathrm{~ms}$ from 0 to $200 \mathrm{~ms}$, then every $50 \mathrm{~ms}$. Sometimes progression was made in $5 \mathrm{~ms}$ steps in order to study more carefully certain critical periods. At each delay, 10 amplitudes of the conditioned $\mathbf{H}$ response were measured peak to peak and compared with 10 unconditioned values. The $F$ responses of the tibialis anterior were rectified and integrated. The amplitude variations of the conditioned responses (expressed as a percentage of reference values) were plotted on the ordinate of a graph, the abscissa of which consisted of the delay in $\mathrm{ms}$ between the stimulation of the sural nerve and that of the posterior tibial or lateral popliteal nerves. A semilogarithmic scale was usually employed. In a single experimental session, which never exceeded 3 hours, it was usually possible to complete two excitability curves.

\section{Results}

\section{A CONDITIONING OF THE SOLEUS H REFLEX}

\section{Ipsilateral stimulation}

Figure 1 illustrates an example of the variations in amplitude of the monosynaptic reflex of the soleus (Hoffmann reflex) following stimulation of the ipsilateral sural nerve. The test reflex had an amplitude of $\mathrm{H} \mathrm{Max} / 2$, and sural stimulation evoked a feeble tactile sensation $(1.5 \times$ threshold $)$. During the first $40 \mathrm{~ms}$, the amplitude of the $\mathrm{H}$ reflex remained similar to control values. Subsequently, there was facilitation which attained its maximum between 60 and $70 \mathrm{~ms}$. Then the amplitude of the conditioned reflex fell to $95 \%$ of control values after $100 \mathrm{~ms}$. After this there was a new facilitation which was maximal after $150 \mathrm{~ms}$ and had disappeared after $200 \mathrm{~ms}$. A similar time course was seen in all cases, exhibiting two 


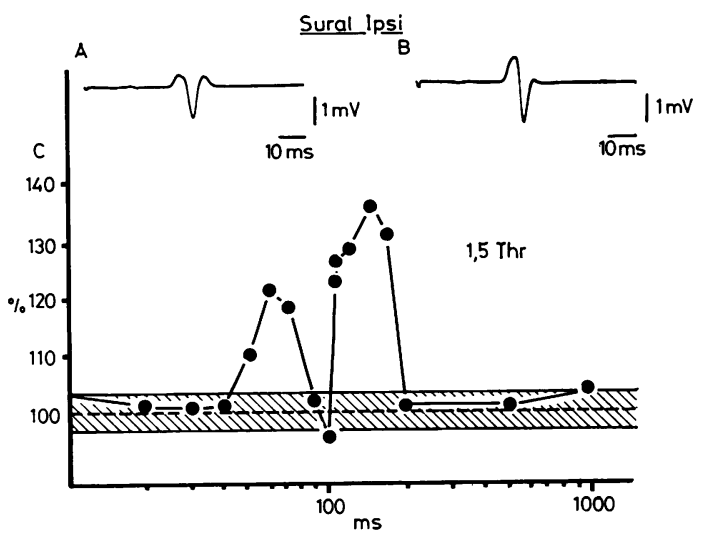

Fig 1 A: An example of a control $H$ reflex. $B$ : The reflex observed 120 msec after ipsilateral sural nerve stimulation is enhanced. C: Recovery curve of the soleus $H$ reflex after stimulation of the ipsilateral sural nerve. Test reflex: $H \mathrm{Max} / 2$. The intensity of stimulation $=1.5 \times$ tactile threshold intensity. In abcissa (semi log): delays in ms between the stimulation of the sural nerve and that of the posterior tibial nerve. In ordinate, the amplitudes of the conditioned responses expressed as a percentage of reference values. The standard deviations are indicated on both sides of the control values.

peaks of facilitation which we will call $F(a)$ and F(b).

Changes in the intensity of stimulation

Figure 2 (A, B, C) illustrates the influence of an increase in the intensity of sural stimulation in a single subject. In $\mathrm{A}$, the stimulus intensity was just above sensation threhold. The effects observed were weak, and amplitude changes did not exceed $10 \%$. However, two periods of facilitation were distinguished, corresponding in time to $F(a)$ and $\mathrm{F}(\mathrm{b})$ in fig 1 .

In $B$, sural stimulation was at $2.5 \times$ threshold, giving rise to distinct tactile sensation. Between 20 and $25 \mathrm{~ms}$ after the beginning of stimulation there appeared an inhibition which increased up to $40 \mathrm{~ms}$. Then the amplitude again increased progressively to reach a facilitation level of $133 \%$ after $80 \mathrm{~ms}$, that is after the same time interval as $F(a)$. The facilitation then diminished, and after about $110 \mathrm{~ms}$ was at control levels. Then a new facilitation appeared which culminated in values of $130 \%$ after $140 \mathrm{~ms}$. This delay corresponds to $F(b)$ in fig 1. Finally, there was a rapid decrement and conditioned values again became smaller than reference values between 170 and $500 \mathrm{~ms}$.

In $C$, the stimulus was at $4.5 \times$ threshold, and
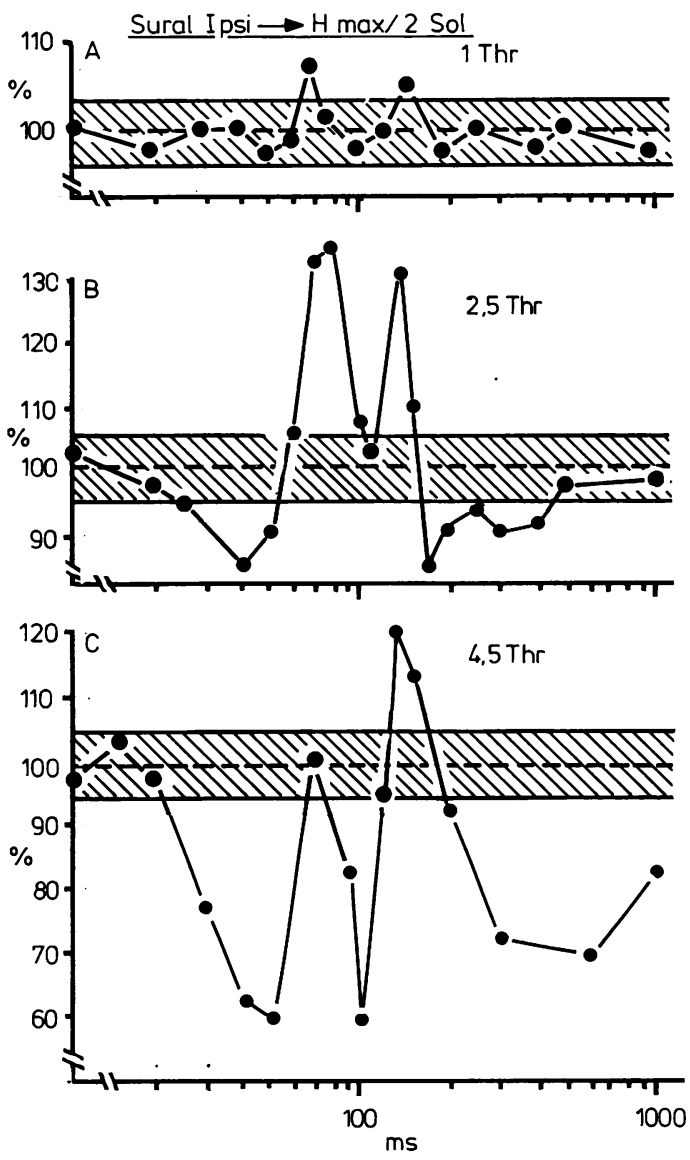

Fig 2 Recovery curves of the soleus $H$ reflex (H Max/2) after stimulation of the ipsilateral sural nerve at different intensities. $A$ : at threshold; $B$ : at $2.5 \times$ threshold intensity and $C$ : at $4.5 \times$ the threshold intensity. In abcissa (semi log): delays in $m s$ between the stimulation of the sural nerve and that of the posterior tibial nerve. In ordinate, the amplitude of the conditioned responses expressed as a percentage of reference values. The limits of standard deviations appear on both sides of the control values.

evoked a painful sensation. The initial inhibition was more marked than in B. At a delay corresponding to $F(a)$, the amplitude of the conditioned reflex increased, and at $70 \mathrm{~ms}$ conditioned amplitudes were equal to controls. Subsequently the inhibition was reinforced over some tens of milliseconds, and at a latency corresponding to $\mathrm{F}(\mathrm{b})$, there appeared a facilitation of $20 \%$. Later, the conditioned reflex was again inhibited, and this 
inhibition lasted beyond $1000 \mathrm{~ms}$. The curve obtained on painful stimulation (fig 2C) appeared clearly to result from a background of intense and prolonged inhibition on which supervened, at the same latencies as $F(a)$ and $F(b)$, two phases of disinhibition and/or facilitation.

With similar intensities of stimulation, on several occasions in the same subject, moderate variations in the excitability curves were observed. On the other hand, in different subjects the differences were more marked. These differences were mainly in intensity; sometimes in latency and duration of the phases of facilitation when the stimulus intensity was at tactile level, and in intensity and duration of inhibition when the stimulus was painful. Thus in some subjects the facilitation shown in fig 2C did not appear, and there was merely a disinhibition of the conditioned reflex at latencies corresponding to $F(a)$ and $F(b)$. When the intensity of stimulation was just above threshold, it was never possible to observe a single peak of facilitation; the two peaks always appeared together.

Following tactile stimulation, $F(a)$ reached its maximum after an average of 81 with SD of $\pm 7.2 \mathrm{~ms}$ and showed a mean facilitation of 138 (SD $\pm 22 \%$ ) (29 curves). When the stimulus was painful, the peak latency of $F(a)$, supervening on a background of inhibition, was reduced to 70 $(\mathrm{SD} \pm 8.2 \mathrm{~ms})$, but the facilitation had practically disappeared $(102 \%$ of control). In the same 29 curves, following stimulation at tactile intensity, $\mathrm{F}(\mathrm{b})$ reached its maximal amplitude $(133 \cdot 5 \pm 19 \%)$ after $148 \pm 17 \mathrm{~ms}$. When stimulation was of painful intensity, the peak latency of $F(b)$ was at $140 \pm 15 \mathrm{~ms}$ and the facilitation at $124 \pm 21$. Under any conditions of stimulation, the duration of $F(a)$ (mean $52.7 \pm 5.2 \mathrm{~ms}$ ) was shorter than that of F(b) $(125 \pm 19 \mathrm{~ms})$. We were unable to establish any correlation between the height of the subjects and the variations in latency of the $F(a)$ and $F(b)$ peaks.

\section{Stimulus duration}

The number of shocks applied to the sural nerve was varied and curves drawn for the same subjects following 1, 2, 7 and 12 conditioning shocks of $1 \mathrm{~ms}$ (frequency of $300 \mathrm{~Hz}$ ). The intensity of a single shock, eventually repeated in a train, was fixed at $2 \times$ tactile threshold. Increase in the number of shocks changed the sensation of touch, which became painful with 12 shocks. An increase in the number of conditioning shocks gave rise to an increase in the initial inhibition and its earlier appearance. On the other hand, the $F(a)$ phase began at the same latency while the $F(b)$ phase was of shorter duration; however, the peak appeared at the same latency. This reduction in the duration of $F(b)$ was related to an increase in the inhibitory phase which appeared between the two phases of facilitation.

\section{Contralateral stimulation}

Figure 3 (taken from the same subject as fig 2) illustrates changes in amplitude of the $\mathrm{H}$ reflex (value of $\mathrm{H} \mathrm{Max} / 2$ ) following stimulation of the contralateral sural nerve. The results shown in the
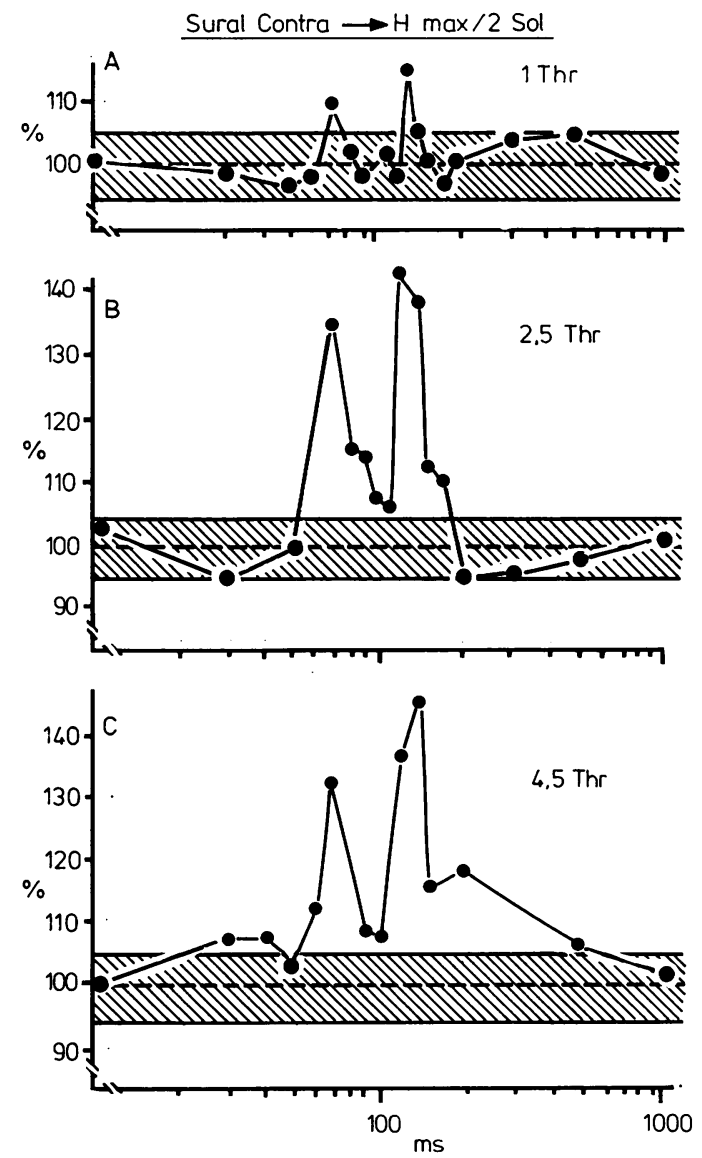

Fig 3 Recovery curves of the soleus $H$ reflex after stimulation of the contralateral sural nerve at different intensities. A: at threshold intensity; B: at $2.5 \times$ threshold intensity and $C:$ at $4.5 \times$ threshold intensity. Co-ordinates as in figs 1 and 2. The curves of this figure and of fig 2 were obtained from the same subjects at two different experimental sessions. The limits of standard deviations appear on both sides of the control values. 
figure were obtained during a single experimental session. Curve A corresponds to stimulation at threshold. Reflex amplitude was little changed, but was facilitated at 70 and $140 \mathrm{~ms}$. Curve B represents a stimulus eliciting a tactile sensation. Two well-marked peaks of facilitation are seen, the first at $70 \mathrm{~ms}$ and the second at $140 \mathrm{~ms}$. This curve is very similar to that illustrated in fig 1 following ipsilateral stimulation of the same intensity, complete with phases $F(a)$ and $F(b)$. Curve $C$ was obtained following frankly painful stimulation. In this case, no early inhibition was seen, thus clearly distinguishing it from similar ipsilateral stimulation (fig 2C). On the contrary, there seemed
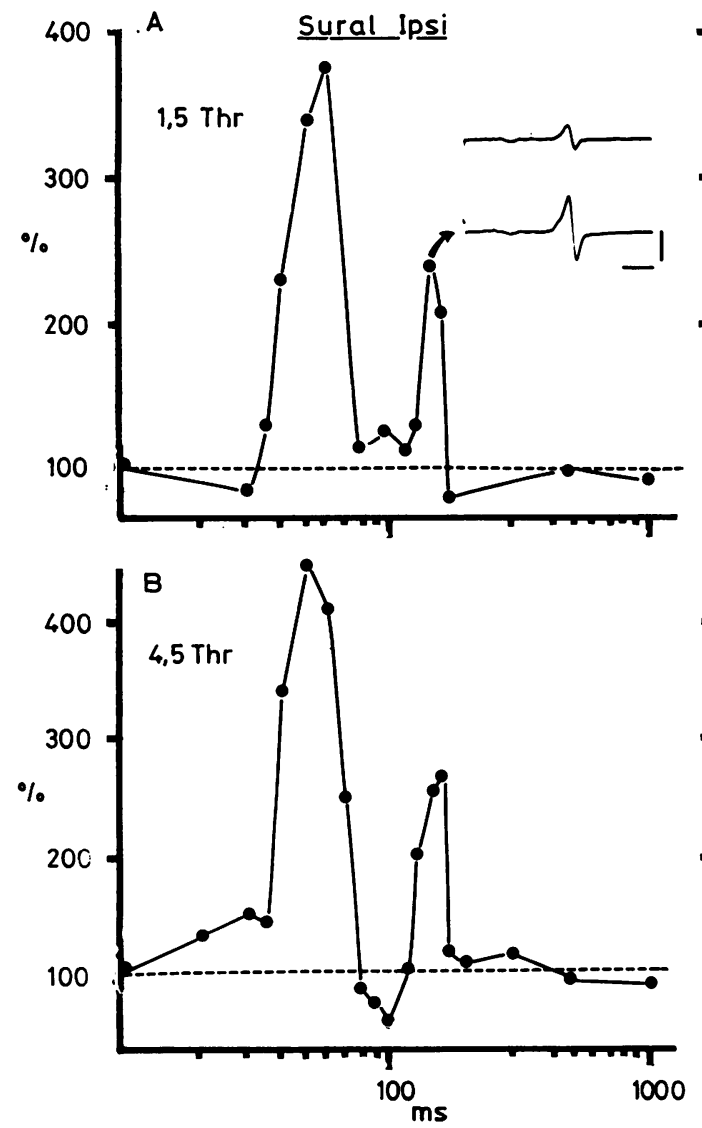

in the case of contralateral stimulation to be a slight facilitation of $10 \%$ at the latency where inhibition was observed on ipsilateral stimulation. There were two peaks of facilitation $F(a)$ and $F(b)$ falling at the same time intervals as with less intense stimuli. In six subjects, recordings following ipsilateral and contralateral stimulation of the same intensity were made during the same experimental session. The facilitation peaks fell at the same latencies.

\section{B CONDITIONING OF THE TIBIALIS ANTERIOR MOTONEURONE POOL}

The excitability of this motoneurone pool was less
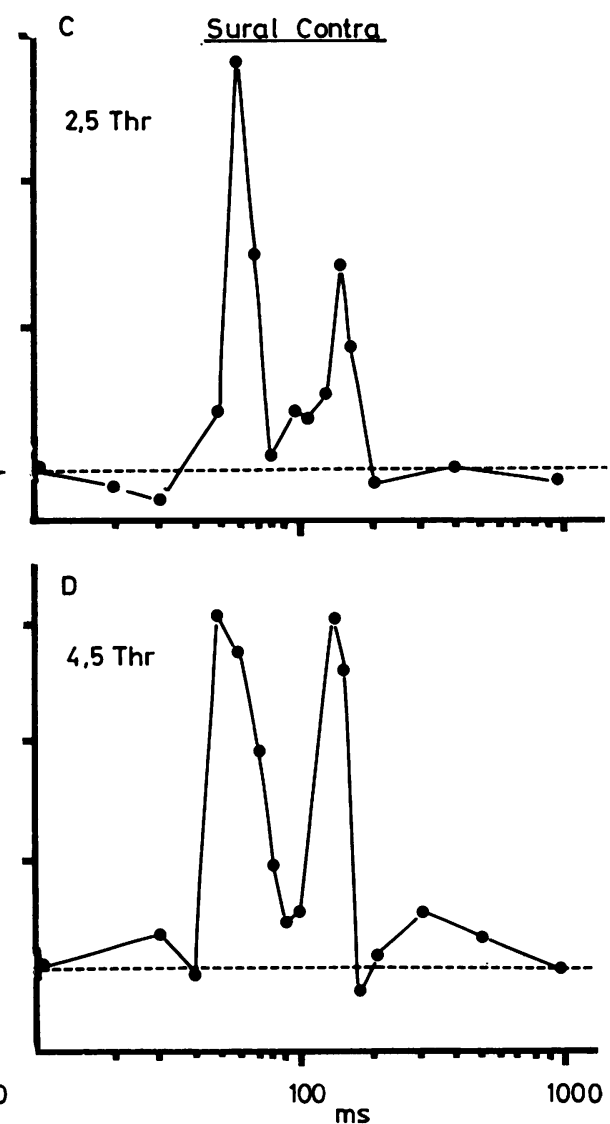

Fig 4 Recovery curves of the tibial anterior $H$ reflex after stimulation of the ipsilateral (on the left) and the contralateral (on the right) sural nerves. Abcissa (semi log): delays in ms between the stimulation of the sural nerve and that of the peroneal nerve at the head of the fibula. Ordinate: the amplitudes of the conditioned responses expressed as a perecentage of the reference values. Note that the high values of facilitations are due to the low level of the reference values. In $A$, the conditioning sural nerve intensity equals $1.5 \times$ threshold intensity; in insert, examples of reflexes recorded from the tibialis anterior: upper: control; lower: conditioned at a latency of $150 \mathrm{~ms}$ (arrow). Calibration: $100 \mu \mathrm{V}$ and $10 \mathrm{~ms}$. In B, 4.5 $\times$ threshold intensity. In C (contralateral stimulation), the stimulation intensity is $2.5 \times$ threshold and in $D$, $4 \cdot 5 \times$ threshold. 


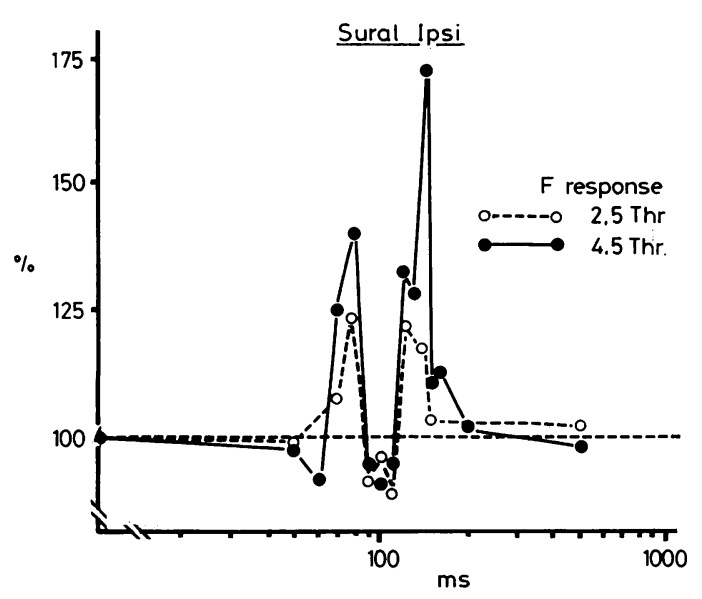

Fig 5 Recovery curves of the tibial anterior $F$ responses after stimulation of the ipsilateral sural nerve. Interrupted line: conditioning stimulation intensity: $2.5 \times$ threshold intensity; continuous line: $4.5 \times$ threshold intensity. Co-ordinates: as in preceding figures.

easy to test than that of the soleus. A Hoffmann reflex, of small amplitude, was found in seven subjects. The ratio of $\mathrm{H} \mathrm{Max} / \mathrm{M}$ Max is from 2 to $4 \%$; the latency was the same as for the soleus $\mathrm{H}$ reflex. Other subjects exhibited a well developed $F$ response. Either of these responses was used to explore the excitability of the tibialis anterior motoneurone pool following ipsilateral or contralateral sural nerve stimulation.

\section{Ipsilateral stimulation}

A low intensity stimulus (fig 4A) did not alter the amplitude of the $\mathbf{H}$ reflex before $30 \mathrm{~ms}$. After this, there was a facilitation which reached its maximum at $60 \mathrm{~ms}$. Subsequently, between 90 and $110 \mathrm{~ms}$, the amplitude returned to control values. Another, less well-marked, facilitation then developed culminating at $15 \mathrm{~ms}$ and disappearing

Table Contralateral sural nerve stimulation

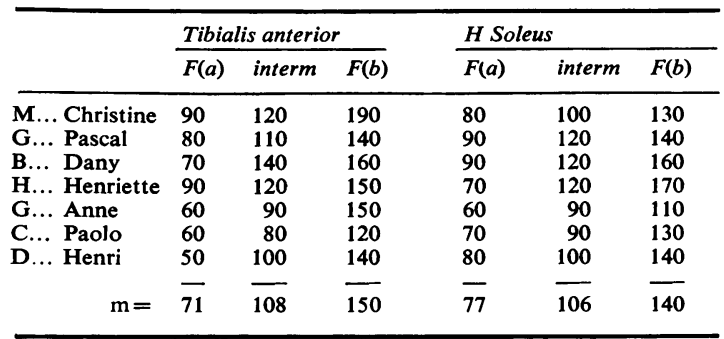

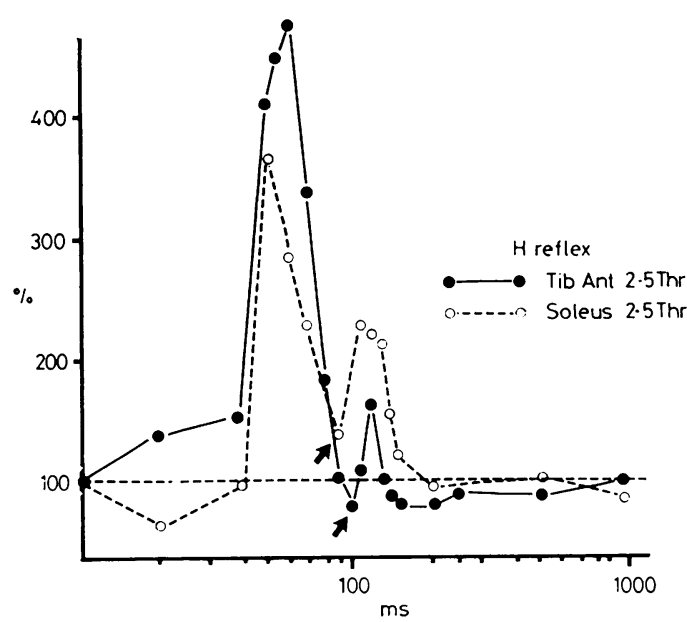

Fig 6 Comparison of soleus and tibial anterior $H$ reflexes recovery curves after the same conditioning (contralateral sural nerve stimulation at $2.5 \times$ threshold intensity) in one given subject. Co-ordinates: as in preceding figures. In both curves, two peaks can be identified occurring at the same latencies. The intermediate point is indicated by an arrow.

after $180 \mathrm{~ms}$. When the intensity of stimulation was increased (fig 4B), an early facilitation of moderate degree was seen after $20 \mathrm{~ms}$, with a peak of facilitation following between 30 and $80 \mathrm{~ms}$. Subsequently, there was a $20 \%$ inhibition lasting for $40 \mathrm{~ms}$. A second phase of facilitation followed, with its maximum at $150 \mathrm{~ms}$. From $200 \mathrm{~ms}$ onwards, amplitude returned to pre-conditioning levels. It can be seen that the two peaks of facilitation fell at latencies very similar to those of $F(a)$ and $F(b)$ as described above. The amplitude of the $F$ response developed in the same way as that of the $\mathrm{H}$ reflex illustrated in fig 5 . In fact there were two well-differentiated phases of facilitation; they were more evident when the stimulus became painful.

\section{Contralateral stimulation}

Stimuli of medium $(2.5 \times$ threshold) or high ( 4.5 $\times$ threshold) intensity elicited two peaks of facilitation at latencies between 50 and $60 \mathrm{~ms}$ for the first and 130 to $15 \mathrm{~ms}$ for the second. Intraindividual variations, as for the soleus muscle, were small, while those between subjects were more marked, as can be seen in the table. In the table, a comparison was made between the latencies of the facilitation peaks $F(a)$ and $F(b)$ for the tibialis anterior and the soleus following stimulation of the contralateral sural nerve. In addition, to see 
whether the curves develop in phase with one another, the latency of the lowest amplitude (intermediate point) between the two peaks was measured. On average, the first peak appeared $6 \mathrm{~ms}$ earlier in the tibialis anterior than in the soleus. The latencies of the intermediate point were very close to one another, while the second peak was $10 \mathrm{~ms}$ earlier in soleus. Thus there were only slight differences, indicating that facilitation occurred in phase in the two antagonistic motoneurone pools represented by the tibialis anterior and soleus. This was confirmed by fig 6 which shows an illustrative example.

\section{Discussion}

One general point emerges from our results: sural stimulation elicits not one but two distinct phases of facilitation, which we have called $F(a)$ and $F(b)$. Two peaks appear, whatever the intensity of stimulation employed, the only exception being that sometimes painful ipsilateral stimulation gives rise only to a simple disinhibition. This double facilitation is not limited to the ipsilateral soleus, since it is also seen after contralateral conditioning stimulation, with quantitatively comparable results. It is not even limited to foot extensors since excitability curves for the motoneurone pool of tibialis anterior exhibit the same biphasic facilitation occuring at the same latencies.

Confining our consideration to the excitability curves for the soleus, it may be thought that there are not in fact two separate facilitations depending on two different mechanisms, but a single facilitation interrupted by activation of the antagonist motoneurone pool according to the established rules of reciprocal innervation. But if excitability changes in the motoneurone pools of both soleus and tibialis anterior are considered together (compare figs 2 and 4 , and 3 and 6 , and the table) it can be seen that the facilitations are strictly synchronous with one another, as are the lower amplitude events between the peaks. Such results make it possible to exclude the possibility of a single phase of facilitation interrupted by the activation of an antagonist motoneurone pool. Despite earlier investigations on the influence of sural stimulation on the excitability of the soleus motoneurone pool, biphasic facilitation has not been reported as a systematic phenomenon, though it has been incidentally mentioned by Gassel and Ott. ${ }^{11}$

The effects of ipsilateral sural stimulation on the soleus $H$ reflex have been investigated by Bathien and Hugon, ${ }^{20}$ Hugon, ${ }^{9}$ Gassel, ${ }^{5}$ Gassel and Ott, ${ }^{11}$ and Castaigne et al; ${ }^{21}$ Hugon $^{9}$ also studied the effects of contralateral stimulation. The results reported by these various authors are not all in agreement. Bathien and Hugon ${ }^{20}$ observed monophasic curves for both the Hoffmann and tendon reflexes exhibiting a strongly marked inhibition, maximal $100 \mathrm{~ms}$ after ipsilateral non-painful stimulation. Hugon ${ }^{9}$ described the same inhibitory phenomenon following painful stimulation, while reporting facilitation after non-painful stimulation. On painful stimulation of the contralateral sural nerve, the same author observed facilitation at $200 \mathrm{~ms}$; he did not observe two distinct peaks of facilitation although he took into consideration the influence of the intensity of the conditioning stimulus. Gassel and Ott ${ }^{11}$ studied recovery curves of the soleus tendon reflex following cutaneous stimuli applied respectively to the dorsal and plantar surfaces of the ipsilateral foot. They described two changes in excitability: one early, between 40 and $90 \mathrm{~ms}$, the other later between 110 and $250 \mathrm{~ms}$. The first phase indicates either facilitation or inhibition, according to the site of cutaneous stimulation. Unlike Hugon, these authors did not systematically explore variations in the intensity of stimulation.

Gassel et $a l^{21}$ described intense inhibition of the ipsilateral $\mathrm{H}$ reflex, maximal at $70 \mathrm{~ms}$ and disappearing at about $100 \mathrm{~ms}$, following painful stimulation of the little toe. The influence of sural stimulation on the tibialis anterior motoneurone pool has not been described hitherto, although Hugon $^{9}$ studied the effects on the short head of biceps.

The demonstration of the two peaks $F(a)$ and $F(b)$ of facilitation in our study is doubtless related to the technical conditions employed, which involved the investigation of excitability by repeated tests at very brief intervals, sometimes less than $10 \mathrm{~ms}$; the existence of these two peaks is an unexpected phenomenon. However, certain comparisons can be made with these results. It is well known that in man motor activity elicited by exteroceptive stimulation exhibits two distinct phases, in the case of lower limb flexion reflexes, ${ }^{4}$ of the short biceps reflex, ${ }^{9}$ upper limb flexion reflexes $^{22}$ and modulation of flexor EMG. ${ }^{8}$ The appearance of activity simultaneously in antagonistic muscles such as tibialis anterior and soleus does not appear to obey the classical rules of reciprocal innervation. However, our results also demonstrate the existence of reciprocal innervation. This appears when consideration is given to the curves obtained after ipsilateral painful stimulation. In that particular situation, a background 
of inhibition of the soleus $\mathrm{H}$ reflex is observed (fig 2C); it begins early, after $20 \mathrm{~ms}$, and lasts more than a second. In the tibialis anterior motoneurone pool, the same stimulus, instead of causing an inhibition, evokes a slight facilitation which appears after a short latency (fig 4B). Thus in our results, a distinction must be drawn between a general phenomenon, independent of the side of stimulation or of the motoneurone pools involved, represented by the two peaks of facilitation; and a more particular phenomenon, obeying the rules of reciprocal innervation, represented by the inhibition engendered in soleus by ipsilateral noxious stimulation and mirrored by facilitation of tibialis anterior. It was also seen, particularly in the curve shown in fig $2 \mathrm{C}$, that the facilitation was sometimes replaced by a disinhibition. Given the similarity of the latencies and general appearance of the phenomena, we have classed these two together.

With respect to crossed phenomena, Robinson et $a l^{2}$ have shown that the excitability curve of the soleus $\mathrm{H}$ reflex following conditioning evocation of another $\mathbf{H}$ reflex indicates the prevalence of inhibition after ipsilateral stimulation while contralateral stimulation elicits only facilitation. These recall mirror phenomena.

The neurophysiological mechanisms of the part of the inhibition which exhibits a reciprocal nature, and the peaks of facilitation which appear not to obey this rule, merit separate discussion:

\section{Ipsilateral inhibition}

The human sural nerve ${ }^{23}$ contains essentially two types of fibre. The first, belonging to group II (A beta), is formed by axons whose diameter lies between 9 and $13 \mu \mathrm{m}$; the second, belonging to group III (A delta), has axons with diameters between 3 and $6 \mu \mathrm{m}$. There are few $C$ fibres, and to activate them requires intensities of stimulation which have not been used in this study. Conduction velocities of group II and III fibres are from 30 to $55 \mathrm{~m} / \mathrm{s}$ and 7.5 to $30 \mathrm{~ms} / \mathrm{s}$. The distance from the ankle to T12-L1 being about 1 metre, impulses in group II fibres arrive at the spinal cord with a minimum latency of $18.1 \mathrm{~ms}$, while those in slower fibres arrive after $34 \mathrm{~ms}$ or later. Since the inhibition sometimes appears within $20 \mathrm{~ms}$, group III fibres cannot be involved. Group II fibres, on the other hand, are a component of the flexor reflex afferents (FRA), and may activate the soleus motor nucleus by means of interneurones, perhaps IA. However, such a mechanism cannot account for the long duration of the inhibition. Furthermore, it is unlikely that there is a single mech- anism of inhibition; different components may either sum or succeed one another. Thus presynaptic inhibition exerted by cutaneous fibres on IA axons may be involved, ${ }^{24}$ and later impulses transmitted by group III fibres. Finally, an inhibitory mechanism of the type described by Shimamura et al $^{25}$ acting via a spino-bulbo-spinal circuit, may be involved. However, purely spinal mechanisms cannot be excluded since inhibitory phenomena lasting for several seconds have been demonstrated following exteroceptive stimulation in patients with spinal transection. ${ }^{4}$ It may be that long lasting transmitters should henceforth be considered in the interpretation of long lasting inhibition.

\section{Facilitation peaks $F(a)$ and $F(b)$}

Gassel and Ott $^{11}$ described two phases of excitability change in soleus and attributed them to the desynchronisation of messages, particularly those in groups II and III, arriving at the spinal cord. This hypothesis is however unlikely because even low-intensity conditioning stimuli (figs $2 \mathrm{~A}$ and $3 \mathrm{~A}$ ), which are unlikely to activate group III fibres, cause appearance of the two peaks. It may therefore be concluded that both peaks of facilitation, after either ipsilateral or contralateral stimulation, are related only to group II (A beta) afferents. The suggestion may therefore be made that the two peaks are related to separate central processing of a single afferent message. Segmental spinal interneuronal circuits may be involved in this mechanism. However, this is difficult to envisage, since it does not take account of the effects observed after contralateral stimulation; it is believed-at least in the cat-that A beta primary afferents are never involved in crossed effects. ${ }^{26}$ Non-segmental neuronal mechanisms must therefore be considered. The latencies of the peaks are compatible with a supraspinal effect, since they appear at more or less the same latencies as the activities attributed by Marsden $e t a l^{27}$ to transcortical reflexes involving the soleus. Further experiments are necessary to determine the circuits responsible for the transmission of the two waves of facilitation. The results of such research are described in a succeeding article.

\section{References}

1 Paillard J. Réflexes et régulations d'origine proprioceptive chez l'homme. Paris: Arnette 1975; 293.

2 Robinson KL, McIlwain JS, Hayes KC. Effects of H-reflex conditioning upon the contralateral 
alpha motoneuron pool. Electroencephalogr Clin Neurophysiol 1979; 46:65-71.

3 Bathien N, Bourdarias $\mathrm{H}$. Lower limb cutaenous reflexes in hemiplegia. Brain 1972; 95:447-56.

4 Shahani BT, Young RR. Human flexor reflexes. J Neurol Neurosurg Psychiatry 1971; 34:616-27.

5 Gassel MM. The role of skin areas adjacent to extensor muscles in motor neurone excitability: evidence bearing on the physiology of Babinski's response. J Neurol Neurosurg Psychiatry 1970; 33:121-6.

6 Jenner JR, Stephens JA. Evidence for a transcortical cutaneous reflex response in man. $J$ Physiol (Lond) 1979; 39-40.

7 Meier-Ewert K, Schmidt C, Nordmann G, Humme U, Dahm J. Averaged muscle-response to repetitive sensory stimuli. In: JE Desmedt, ed. New Developments in Electromyography and Clinical Neurophysiology. Basel: Karger 1973, vol 3, 767-72.

8 Piesiur-Strehlow B, Meinck HM. Response patterns of human lumbosacral motoneurone pools to distant somatosensory stimuli. Electroencephalogr Clin Neurophysiol 1980; 48:673-86.

9 Hugon M. Réflexes polysynaptiques cutanés et commandes voluntaires. Thèse de Doctorat d'Etat ès Sciences Naturelles. Faculté des Sciences de Paris 1967.

10 Pierrot-Deseilligny E. Morin C, Bergego C, Tankov N. Pattern of Group I fibre projections from ankle flexors and extensors muscles in man. Exp Brain Res 1981 (in press).

11 Gassel MM, Ott KH. Local sign and late effects on motoneuron excitability of cutaneous stimulation in man. Brain 1970; 93:95-106.

12 Bawa $P$, Tatton WG. Motor unit responses in muscles stretched by imposed displacements of the monkey wrist. Exp Brain Res 1979; 37:417-37.

13 Chan CWY, Melvill Jones G, Kearney RE, Watt DGD. The "late" electromyographic response to limb displacement in man. I. Evidence for supraspinal contribution. Electroencephalogr Clin Neurophysiol 1979; 46:173-81.

14 Gottlieb GL, Agarwal GC. Response to sudden torques about ankle in man: myotatic reflex. $J$ Neurophysiol 1979; 42:91-106.

15 Lee RG, Tatton WG. Long-loop reflexes in humans: clinical application. In: Desmedt JE, ed.
Progress in Clinical Neurophysiology. Basel: Karger 1978, vol 4, 320-33.

16 Marsden CD, Merton PA, Morton HB. Servo action in human voluntary movement. Nature 1972; 238:140-3.

17 Fisher MA, Shahani BT, Young RR. Assessing segmental excitability after acute rostral lesions. Neurology (Minneap) 1978; 28:1265-71.

18 Schiller HH, Stalberg E. F responses studied with single fibre EMG in normal subjects and spastic patients. J Neurol Neurosurg Psychiatry 1978; 41:45-53.

19 Desmedt JE. Human reflexes. Pathophysiology of Motor Systems. Methodology of Human Reflexes. In: Desmedt JE, ed. New Developments in Electromyography and Clinical Neurophysiology. Basel: Karger 1973, vol 3; 870.

20 Bathien N, Hugon M. Etude chez l'homme de la dépression d'un réflexe monosynaptique par stimulation d'un nerf cutané. J Physiol (Lond) 1964; 56:285-6.

21 Castaigne JP, Held JP, Cathala HP, PierrotDeseilligny E, Bussel B, Morin C. Modifications de l'excitabilité médullaire induites par une stimulation cutanée chez différentes catégories d'hémiplégiques. Rev Neurol (Paris) 1972; 126: 393-400.

22 Cambier J, Dehen H, Bathien N. Upper limb cutaneous polysynaptic reflexes. J Neurol Sci 1974; 22:39-49.

23 O'Sullivan DJ, Swallow M. The fibre and content of the radial and sural nerve. J Neurol Neurosurg Psychiatry 1968; 31:464-70.

24 Rudomin $\mathbf{P}$, Dutton $\mathrm{H}$. Effects of conditioning afferent volleys on variability of monosynaptic responses of extensor motoneurons. $J$ Neurophysiol 1969; 32:2-140.

25 Shimamura M, Mori S, Yamauchi T. Effects of spino-bulbo-spinal reflex volleys on extensor motoneurons of hindlimb in cats. $J$ Neurophysiol 1967; 29:319-32.

26 Rosenberg ME. Synaptic connexions of alpha extensor motoneurons with ipsilateral and contralateral cutaneous nerves. J Physiol (Lond) 1970; 207:231-55.

27 Marsden CD, Merton PA, Morton HB. Stretch reflex and servo action in a variety of human muscles. J Physiol 1976; 259:531-60. 